\title{
1957 \\ POWDER METALLURGY \\ SYMPOSIUM
}

$\boldsymbol{T}$

HE symposium on powder metallurgy which is published on the following pages was arranged by the Powder Metallurgy Committee of the Institute of Metals Division and presented at the AIME Annual Meeting in New Orleans on Feb. 28, 1957.

One of the principal purposes of this symposium was to acquaint engineers in the southwestern part of the country with the techniques of powder metallurgy. The subjects of the first two papers were, therefore, chosen because of their particular interest to engineers in the aircraft and petroleum industries. Filters made from metal powders are widely used both in airplanes and engines. For drilling wells, the use of cemented carbides and impregnated diamond drilling bits, important products of the powder metallurgy industry, need hardly be emphasized.

These two individual papers are followed by a panel discussion on high strength structural parts. Fabricating structural parts from metal powders lifted powder metallurgy beyond the limits of a technique used only for rather specialized applications, and put it squarely into competition with other, more conventional methods of fabricating machine parts, such as machined castings or steel bar stock, diecasting, forging, etc. Powder metallurgy as a technique for making such parts was, at first, limited to applications where only moderate mechanical properties, comparable to those of a medium grade of cast iron, were required. Raising the level of mechanical properties of these parts by techniques which have been recently developed has opened up large numbers of potential new applications to the powder metallurgy technique. A mere beginning has been made in actually exploiting these applications. The panel discussion was therefore designed to acquaint engineers with the new possibilities in powder metallurgy.

-F. V. Lenel 